\title{
FATORES ASSOCIADOS À DEPRESSÃO PÓS-PARTO: Revisão Integrativa
}

\author{
Aline Sharlon Maciel Batista Ramos ${ }^{1}$; Ana Caroline Queiroz Martins ${ }^{2}$; Débora Luana \\ Ribeiro Pessoa ${ }^{3}$; Márcia Cristina Aguiar Mendes Machado; Francisca Maria Ferreira \\ Noronha $^{5}$
}

1 Enfermeira, Doutoranda em Ciências da Saúde (UERJ). Professora Assistente da Universidade CEUMA, Brasil. E-mail: alinesharlon@gmail.com

2 Discente do $5^{\circ}$ ano do Curso de Enfermagem da Universidade CEUMA. E-mail: carollijesus@hotmail.com

3 Farmacêutica, Doutorado em Biotecnologia. Professora Adjunta da Universidade Federal do Maranhão, Brasil. E-mail: debbyeluna2@yahoo.com.br

4 Enfermeira, Mestre em Biologia Parasitária (CEUMA). Professora Assistente da

Universidade CEUMA, Brasil. E-mail:marcia.machado@ceuma.br

5 Enfermeira, Mestre em Biologia Parasitária (CEUMA). Professora Assistente da

Universidade CEUMA, Brasil. E-mail: francisca.noronha@ceuma.br

\section{Recebido em: 06/04/2018 - Aprovado em: 10/06/2018 - Publicado em: 20/06/2018 DOI: 10.18677/EnciBio_2018A100}

\begin{abstract}
RESUMO
A Depressão pós-parto possui alta prevalência no mundo, pois no puerpério os eventos naturais somados à propensão psicológica e psicossocial dão condições para o aumento da vulnerabilidade, deixando a mulher fragilizada e suscetível ao surgimento desse distúrbio. Objetivo: identificar os fatores determinantes para o surgimento de depressão em gestantes no período pós-parto. Material e Métodos: foi realizada uma revisão crítica das publicações na PUBMED referidos aos anos de 2007 a 2017, utilizando-se termos e descritores relacionados à Depressão PósParto. Foram excluídos os artigos que não atenderam aos critérios de inclusão, aqueles que apresentaram duplicidade de dados e os artigos que após leitura pormenorizada não atenderam ao objetivo proposto nessa revisão. Resultados: foram identificados, nas amostras dos estudos que utilizaram mulheres nos períodos de gravidez, pós-parto imediato e tardio, com ou sem histórico reprodutivo, dez fatores de risco considerados determinantes para o surgimento da depressão após o parto, sendo eles: insatisfação com a gravidez; história de depressão; abuso sexual e violência doméstica antes, durante ou após a gravidez; maior número de estressores vivenciados ao longo da vida; história de depressão pós-parto; baixo índice de saúde mental; depressão pré-natal; sofrimento emocional durante a gravidez e/ou parto; aleitamento por menos de seis meses e baixo índice de ômega 3. Conclusão: A maior exposição das gestantes, tanto nos períodos pré-parto quanto no pós-parto, aos fatores de risco demonstrados neste estudo, principalmente aos sofrimentos emocionais somados ao maior número de estressores vivenciados e a depressão pré-natal, pode ser determinante para o surgimento da depressão pósparto.
\end{abstract}

PALAVRA-CHAVE: Gravidez. Depressão Pós-Parto. Fatores de risco. 


\title{
FACTORS ASSOCIATED WITH POST-DEPARTMENT DEPRESSION: Integrative Review
}

\begin{abstract}
The postpartum depression has a high prevalence in the world, because in the puerperium natural events added to the psychological and psychosocial propensity give conditions for the increase of vulnerability, leaving the woman fragile and susceptible to the onset of this disorder. Objective: to identify the factors considered determinants for the onset of depression in pregnant women in the postpartum period. Method: a critical review of the publications in the PUBMED referring to the years 2007 to 2017, using descriptors related to Postpartum Depression. We excluded articles that did not meet the inclusion criteria, those that presented data duplication and the articles that after detailed reading did not meet the objective proposed in this review. Results: ten risk factors considered to be determinants for the onset of postpartum depression were identified in the samples that used women in the pregnancy, immediate and late postpartum period, with or without a reproductive history, being: dissatisfaction with pregnancy; history of depression; sexual abuse and domestic violence before, during or after pregnancy; greater number of stressors experienced throughout life; history of postpartum depression; low mental health index; prenatal depression; emotional distress during pregnancy and / or childbirth; breastfeeding for less than six months and low omega 3 index. Conclusion: The greater exposure of pregnant women, both in the pre-delivery and postpartum periods, to the risk factors demonstrated in this study, mainly to the emotional sufferings added to the greater number of stressors and prenatal depression, can be determinant for the onset of postpartum depression.
\end{abstract}

KEYWORDS: Pregnancy. Postpartum Depression. Risk factors.

\section{INTRODUÇÃO}

A gravidez é um evento biologicamente natural, porém um período de importante vulnerabilidade emocional, onde sentimentos diversos são vividos, sendo essa fase uma transição que envolve a necessidade de reestruturação e reajustamento em várias dimensões. Causa mudança de identidade e redefinição de papéis. Mudanças iniciadas na descoberta da gravidez que se estendem além do parto, a depender da vivência de cada mulher (SAVIANI-ZEOTI; PETEAN, 2015).

Segundo Medeiros et al., (2016), mesmo se tratando de um fenômeno natural, deve ser acompanhada por uma equipe multidisciplinar, a fim de diminuir as intercorrências durante o pré-natal, trabalho de parto, parto e puerpério. Pois, uma assistência eficiente pode diminuir o surgimento de problemas reais e potencias, facilitar a identificação dos diagnósticos, planejamento e implementação dos cuidados necessários.

No pós-parto, a mulher pode apresentar maior risco para problemas de ordem física, mental e social. Atingindo também a família e a criança, sendo manifestados, muitas vezes, por: dores/incômodos; medos/preocupações; percalços sexuais; redução da autoestima e do cuidado pessoal; dificuldades interacionais com familiares e/ou com o filho; depressão e outros (TEIXEIRA et al., 2015).

Martinez et al., (2016), afirmam que durante o pós-parto, a condição psiquiátrica mais comum é a depressão pós-parto (DPP), um problema de saúde pública de escala mundial. E que apesar de uma proporção significativa das mães, enquanto usuárias da atenção primária de saúde (APS), apresentem para tal alto 
risco, ainda sim os transtornos depressivos geralmente não são detectados ou tratados, apesar da disponibilidade de tratamentos eficazes.

Este quadro manifesta-se, na maioria dos casos, a partir das primeiras quatro semanas após o parto e atinge, habitualmente, sua intensidade máxima nos seis primeiros meses (GALVÃO et al., 2015).

Muitos fatores podem estar relacionados à DPP, como: histórico de depressão, transtorno mental, ansiedade ou problemas emocionais na gravidez, eventos estressantes, apoio social ou financeiro ausente ou insuficiente. E muitas são as consequências desse episódio, como o comprometimento da relação entre mãe e filho (BRITO et al., 2015).

Segundo a Organização Mundial de Saúde (OMS, 2017), a depressão é a principal causa de problemas de saúde e incapacidade em todo o mundo. É um importante fator de risco para o suicídio e é considerado um transtorno mental comum, caracterizada por tristeza persistente e uma perda de interesse e incapacidade de realizar atividades que a pessoa normalmente gosta e as atividades diárias por 14 dias.

Sendo assim, o objetivo deste estudo foi identificar, através de uma revisão integrativa, os fatores de risco considerados determinantes para o surgimento de depressão em gestantes no período pós-parto.

\section{MATERIAL E MÉTODOS}

Com o intuito de conhecer os estudos envolvendo gravidez, depressão e pós-parto, realizou-se uma revisão integrativa da literatura na base de dados PubMed, nos meses de julho e agosto de 2017. Utilizamos palavras-chave em inglês: "pregnancy" AND e "postpartum" AND "depression", sendo que "risck factores" é um descritor indexado no sistema de Descritores em Ciências da Saúde (DeCS) e "pregnancy" e "post partum" são termos associados ao tema de estudo.

Foram selecionados os seguintes filtros na busca avançada: a primeira etapa dos critérios foi à exclusão dos artigos que não possuíam os resumos disponíveis. Após este procedimento, as próximas fases contaram com a leitura dos títulos e/ou dos resumos. Dessa forma, foram excluídos os artigos que o idioma principal não fosse português e inglês. Após a aplicação dos critérios de inclusão e exclusão acima descritos, realizou-se a leitura minuciosa dos artigos na íntegra.

A análise dos dados possibilitou extrair as seguintes informações dos estudos incluídos, Tabela 1: 1) autoria e ano; 2) país de publicação; 3) método; 4) amostra e 5) instrumentos avaliativos utilizados. Após análise, foram organizados e discutidos em três temas principais: fatores associados à depressão pós-parto; fatores de risco associados à depressão em gestantes nos períodos pré e pós-natal; fatores de risco associados à depressão em gestantes no período pós-parto.

No total, foram encontrados 1760 artigos sobre depressão, fatores de risco e pós-parto na base de dados pesquisada, de acordo com as estratégias de busca traçadas. A Figura 1 apresenta o número de artigos identificados na base de dados escolhidos, a partir das combinações das palavras-chave. A fim de delimitar o número de artigos identificados que se adequavam à necessidade dessa revisão, foram estabelecidos critérios de exclusão demonstrados no Quadro 1.

Dessa forma, apesar do número elevado de publicações encontradas, apenas 15 permaneceram dentro dos critérios de exclusão e inclusão já descrita, 
dos quais após uma leitura pormenorizada somente 4 artigos foram considerados para análise final.

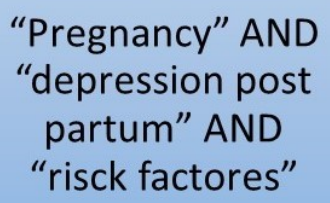

Figura 1. Número de publicações encontradas na base de dados.

\section{Quadro1. Número de publicações por critério na base de dados.}

\section{Total inicial}

Publicação >10 anos

Outras Espécies

Resumos indisponíveis

Outros idiomas

Faixa etária $</>19-44$ anos

Texto completo indisponível

Outras complicações na gravidez

Artigos em duplicidade

Artigos com amostra/instrumentos incompatíveis ao estudo

PubMed
1760
533
192
148
9
76
676
77
2
43

Total para análise 4

\begin{tabular}{|c|c|c|c|c|}
\hline Estudo & Autor/Título/ Ano/ País & Método & Amostra & $\begin{array}{l}\text { Instrumentos } \\
\text { avaliativos utilizados }\end{array}$ \\
\hline E1 & $\begin{array}{l}\text { Gauthreaux et al. } \\
\text { Associação entre a adoção } \\
\text { da gravidez e a } \\
\text { experimentação de de } \\
\text { sintomas de depressão pós- } \\
\text { parto entre novas mães nos } \\
\text { Estados Unidos. } \\
\text { 2017/EUA }\end{array}$ & Transversal & $\begin{array}{l}110.231 \\
\text { mulheres que } \\
\text { deram à luz e } \\
\text { que } \\
\text { completaram } \\
\text { o } \\
\text { Questionário } \\
\text { Essencial da } \\
\text { Fase } 6 \text { da } \\
\text { PRAMS. }\end{array}$ & $\begin{array}{lll}\begin{array}{l}\text { Coleta de amostra na } \\
\text { base de dados }\end{array} & \begin{array}{l}\text { do } \\
\text { Sistema }\end{array} & \text { de } \\
\text { Monitoramento } & \text { de } \\
\text { Avaliação de Riscos de } & \text { de } \\
\text { Gravidez do Centro pra } \\
\text { Controle e Prevenção de } \\
\text { Doenças } & \text { (PRAMS), } \\
\text { Questionário essencial } \\
\text { da fase } 6 \quad \text { da } \\
\text { PRAMS,Testequi- } \\
\text { quadrado e Regressão } \\
\text { logística binária. }\end{array}$ \\
\hline E2 & $\begin{array}{l}\text { Chojenta et al. } \\
\text { Fatores de saúde materna } \\
\text { como riscos para a } \\
\text { depressão pós-natal: um } \\
\text { estudo longitudinal } \\
\text { prospectivo. }\end{array}$ & $\begin{array}{l}\text { Longitudinal } \\
\text { Prospectivo }\end{array}$ & $\begin{array}{l}5.219 \\
\text { mulheres que } \\
\text { deram à luz e } \\
\text { que } \\
\text { completaram } \\
\text { o inquérito } 5 \\
\text { do estudo }\end{array}$ & $\begin{array}{l}\text { Inquérito } 5 \text { do Estudo } \\
\text { Longitudinal Australiano } \\
\text { sobre Saúde da Mulher } \\
(\text { ALSWH). }\end{array}$ \\
\hline
\end{tabular}




\begin{tabular}{|c|c|c|c|c|}
\hline & 2016/ Austrália & & $\begin{array}{l}\text { longitudinal } \\
\text { australiana } \\
\text { sobre saúde } \\
\text { da mulher. }\end{array}$ & \\
\hline E3 & $\begin{array}{l}\text { Turkcapar et al. } \\
\text { Características } \\
\text { sociodemográficas } \\
\text { clínicas da depressão pós- } \\
\text { parto entre as mulheres } \\
\text { turcas: um estudo } \\
\text { prospectivo. } \\
\text { 2015/Turquia }\end{array}$ & Prospectivo & $\begin{array}{l}540 \text { gestantes } \\
\text { (36-40 } \\
\text { semanas de } \\
\text { gestação) }\end{array}$ & \begin{tabular}{lrr}
$\begin{array}{l}\text { Escala de } \\
\text { Depressão }\end{array}$ & \multicolumn{2}{c}{ Ansiedade } \\
Hospitalar \\
(HADS), & Escala de & de \\
Depressão & Pós-natal & de \\
Edimburgo & (EPDS) & e \\
Questionários & \\
sociodemográficos & e \\
clínicos próprios. &
\end{tabular} \\
\hline E4 & $\begin{array}{l}\text { Markhus et al. } \\
\text { O índice de ômega-3 baixo } \\
\text { na gravidez é um fator de } \\
\text { risco biológico para a } \\
\text { depressão pós-parto. } \\
\text { 2013/Noruega }\end{array}$ & $\begin{array}{l}\text { Coorte } \\
\text { Prospectiva }\end{array}$ & $\begin{array}{l}43 \text { mulheres } \\
\text { grávidas de } \\
24 \text { semanas. }\end{array}$ & \begin{tabular}{llr} 
Exames & \multicolumn{2}{c}{ laboratoriais } \\
Escala de & Ansiedade e \\
Depressão & \multicolumn{2}{c}{ Hospitalar } \\
(HADS), Escala de & de \\
Depressão Pós-natal de & Edimburgo (EPDS) e \\
Questionários & \\
sociodemográficos & e \\
clínicos próprios.
\end{tabular} \\
\hline
\end{tabular}




\begin{tabular}{|c|c|c|c|}
\hline & E1, E3 & 1) & Insatisfação com a gravidez; \\
\hline & $\mathrm{E} 1, \mathrm{E} 2$ & 2) & História de depressão; \\
\hline & E1, E3 & 3) & $\begin{array}{l}\text { Abuso sexual e violência doméstica antes, durante ou após } \\
\text { a gravidez; }\end{array}$ \\
\hline \multirow{7}{*}{$\begin{array}{c}\text { FATORES DE RISCO } \\
\text { ASSOCIADOS À } \\
\text { DEPRESSÃO PÓS- } \\
\text { PARTO }\end{array}$} & E1 & 4) & $\begin{array}{l}\text { Maior número de estressores vivenciados ao longo da } \\
\text { vida; }\end{array}$ \\
\hline & E2, E3 & 5) & História de Depressão Pós-parto; \\
\hline & E2 & 6) & Baixo índice de saúde mental; \\
\hline & E3 & 7) & Depressão Pré-Natal; \\
\hline & $\mathrm{E} 2, \mathrm{E} 4$ & 8) & Sofrimento emocional durante a gravidez e/ou parto; \\
\hline & E2 & 9) & Aleitamento por menos de seis meses; \\
\hline & E4 & 10 & Baixo índice de ômega 3. \\
\hline
\end{tabular}

Figura 2. Fatores de risco associados à Depressão Pós-Parto

\section{RESULTADOS E DISCUSSÃO}

Os resultados encontrados foram dezenove fatores de risco para a depressão pós-parto. Porém, após considerar similaridade entre esses achados, consideramos apenas dez fatores de risco, demonstrados na Figura 2, os quais são apresentados e discutidos a seguir:

\subsection{Insatisfação com a Gravidez}

O Estudo (E1) realizado nos Estados Unidos, com uma amostra de 110.231 mulheres e (E3) realizado na Austrália com 5.219 mulheres, concordam que o nível de satisfação em estar grávida, está fortemente associado aos sintomas de DPP.

Gauthreaux et al. (2017), avaliou a relação do desejo de estar grávida com a DPP através dos sentimentos depressivos sentidos e sua frequência. Afirmou que as mulheres que não desejavam a gravidez e as que a desejavam mais tarde, apresentaram em relação às mulheres que desejavam engravidar, maior risco para a DPP. Para Turkcapar et al., também se percebeu essa relação, após identificar em sua amostra, que as mulheres tendo DPP, tinham insatisfação com a gravidez atual. 


\subsection{História de Depressão}

Segundo Gauthreaux et al. (2017), esse achado foi obtido pela avaliação das mulheres categorizadas como gravidez não desejada ou desejada mais tarde, onde através de questionário, o maior percentual de cada categoria relatou ter vivenciado em algum momento da sua vida um episódio depressivo.

De acordo com Chojeta et al. (2016), estudo 2, a história de depressão esteve presente nas mulheres que manifestaram sintomas de DPP, onde de um total de 5.219 mulheres que deram à luz, 820 tiveram a ocorrência de depressão pósparto.

\subsection{Abuso sexual e violência doméstica antes, durante ou após a gravidez}

As evidências de Guathreaux et al. (2017) e Turkcapar et al. (E3), resultantes de questionários aplicados à amostra, concordam que para as mulheres categorizadas como gravidez não desejada ou desejada mais tarde, que relataram abuso sexual e violência doméstica antes ou durante a gravidez, também considerando o seu histórico de reprodução, expressaram maior percentual de ocorrência de sintomas depressivos pós-parto.

\subsection{Maior Número de Estressores Vivenciados ao longo da vida.}

Para Gauthreaux e colaboradores (2017), foram considerados estressores: ter um membro da família doente; separação ou divórcio do parceiro; falta de moradia; a entrevistada ou o cônjuge perderam o emprego; conflitos com o parceiro; cônjuge não desejava gravidez; ser incapaz de pagar as contas; estar em uma luta física; a entrevistada ou o parceiro foram para a prisão; alguém perto da entrevistada usa drogas ou álcool ou alguém perto da entrevistada morreu.

Foram achados para a amostra identificada como gravidez não desejada ou desejada mais tarde, os seguintes resultados, quando: presença de 1 a 2 estressores - aumentou a probabilidade de DPP pela metade; presença de 3 a 5 estressores - duplicou a chance de DPP e a presença de 6 ou mais estressores quadruplicou a possibilidade dessas mulheres desenvolverem DPP.

\subsection{História de Depressão Pós-Parto}

Após levantamento de dados nos períodos envolvendo a gravidez anterior, realizado por Chojeta et al.(2016), foi possível identificar, através de questionários que avaliaram vários possíveis fatores de risco para à DPP já vivenciados, que as mulheres diagnosticadas com DPP na gravidez atual já haviam experimentado esse episódio em alguma gestação anterior, tornando-o recorrente.

Esse achado fortalece o estudo de Turkcapar et al. (2015), que ao avaliar mulheres internadas em um hospital de obstetrícia na Turquia, encontrou nas mulheres que apresentavam sintomas de DPP atual, histórico de depressão pósparto, as quais manifestavam insatisfação com a atual gravidez e pensamentos suicidas durante a gestação.

\subsection{Baixo índice de saúde mental}

Para avaliação de Chojeta et al. (2016), através do questionário SF-36 para avaliar a saúde mental das 5.219 mulheres do estudo, concluiu que as mulheres com sintomas de DPP apresentaram baixo índice de saúde mental, o que diretamente se associou ao bem estar físico e emocional dessas mulheres, que 
também foi avaliado por esse questionário, sendo possível considerar a forte associação entre o baixo nível de saúde mental e a depressão pós-parto.

\subsection{Depressão Pré-Natal}

Existem evidências em todos os estudos analisados sobre a depressão pré-natal, porém somente Turkcapar et al.(2015), considerou em seus resultados que este fator está fortemente relacionado aos sintomas de DPP, através do resultado dos questionários aplicados em sua amostra.

\subsection{Sofrimento emocional durante a gravidez e/ou parto}

O sofrimento emocional durante a gravidez ou no parto foi demonstrado por Chojeta et al. (2016) e Markhus et al. (2013). Questionários aplicados às respectivas amostras expressaram relatos das puérperas sobre os eventos emocionais que envolveram a gestação e o parto. Assim, foi possível concluir que esse fator, quando associado a outros fatores de risco experimentados por elas, pode desenvolver um episódio de depressão no pós-parto.

\subsection{Aleitamento por menos de seis meses}

Para Chojeta et al. (2016), este fator foi considerado um fator materno de risco para a DPP, após analisar no histórico reprodutivo e na gravidez atual, a desistência do aleitamento antes dos seis meses.

Os estudos mostraram que o aleitamento por menos de seis meses afetou a relação da mãe com o bebê, diminuindo a construção do vínculo materno. Concluindo que este fator quando associado a outros fatores de risco já demonstrados nesta revisão, podem interferir no bem estar físico e mental da mulher, contribuindo para o surgimento de um episódio depressivo maior no puerpério (DPP).

\subsection{Baixo índice de ômega-3}

Markhus et al.(2013), realizou um estudo com 43 mulheres grávidas de 24 semanas, sugerindo que o baixo índice de ômega 3 no final da gestação, por volta de 28 semanas, vai interferir na saúde mental da gestante. Visto que, há uma grande demanda de ômega 3 para o feto e que a baixa reposição dietética ou mesmo a não reposição deste, pode comprometer o bem estar mental dessa mãe e por consequência influenciar no surgimento da depressão pós-parto.

Sua amostra foi avaliada através de questionários e de exames laboratoriais, onde conseguiu provar a forte associação entre o baixo nível de ômega 3 no fim da gestação e a presença do episódio depressivo no pós-parto.

\section{CONCLUSÃO}

Concluímos a partir desta revisão que os principais fatores de risco associados à depressão pós-parto são: insatisfação com a gravidez; história de depressão; abuso sexual e violência doméstica antes, durante ou após a gravidez; maior número de estressores vivenciados na vida; história de depressão pós-parto; baixo índice de saúde mental; depressão pré-natal; sofrimento emocional durante a gravidez e/ou parto; aleitamento por menos de seis meses e baixo índice de ômega 3. 
Dentre os fatores de risco encontrados, podemos destacar o histórico de depressão e depressão pré-natal. Eles comprometem a saúde mental da gestante, deixando-a muito mais vulnerável ao desenvolvimento de depressão no pós-parto, já que esta vem ser uma continuidade desses episódios depressivos já experimentados.

Também foi possível perceber que a soma dos sofrimentos emocionais ao maior número de estressores vivenciados na vida pelas mulheres, esteve fortemente associado ao surgimento da DPP. Uma vida emocional desestabilizada pela falta de apoio da família e a instabilidade na relação afetiva com o cônjuge, são os principais estressores que implicam no surgimento de episódios depressivos relacionados à gravidez.

Destacamos como limitador desse estudo a pouca produção em língua portuguesa em relação à depressão pós parto, especialmente no que concerne ao âmbito atenção primária em saúde

Outros fatores de risco destacados nos estudos, como os fatores sociodemográficos( escolaridade; estado civil; ocupação; apoio social e renda) não foram considerados, pois apresentaram contradições nos resultados. Estes refletem a necessidade de avaliação de fatores ambientais e culturais para a implementação de políticas públicas direcionadas à ocorrência de depressão no ciclo gravídicopuerperal.

\section{REFERÊNCIAS}

BRITO, Cynthia Nunes de Oliveira et al . Depressão pós-parto entre mulheres com gravidez não pretendida. Rev. Saúde Pública, São Paulo , v. 49, 33, 2015 . Disponível em <http://www.scielo.br/scielo.php?script=sci_arttext\&pid=S003489102015000100225\&lng=pt\&nrm=iso>. Acesso em: 04 junho 2017. http://dx.doi.org/10.1590/S0034-8910.2015049005257.

GALVÃO, Anna Carolinne Castro et al. Prevalência de depressão pós-parto e fatores associados: uma revisão integrativa. ReOnFacema, 2015 Ago-Out; 1(1):54-58. Disponível

http://www.facema.edu.br/ojs/index.php/ReOnFacema/article/view/3/14>. Acesso em: 24 de Maio de 2017.

MARTINEZ, Pablo; VOHRINGER, Paul A.; ROJAS, Graciela. Barreiras de acesso a tratamento para mães com depressão pós-parto em centros de atenção primária: um modelo preditivo. Rev. Latino-Am. Enfermagem, Ribeirão Preto , v. 24, e2675, $2016 . \quad$ Disponível em <http://www.scielo.br/scielo.php? script=sci_arttext\&pid=S0104-11692016000100312\&lng=pt\&nrm=iso $>$. acessos em 25 abril 2017 Epub 28-Mar-2016. http://dx.doi.org/10.1590/1518-8345.0982.2675.

Organização Mundial de Saúde (OMS). Com depressão no topo da lista de causas de problemas de saúde, OMS lança a campanha "Vamos conversar" 30 de março de 2017. Disponível em: <http://www.paho.org/bra/index.php? option=com_content\&view=article\&id=5385:com-depressao-no-topo-da-lista-decausas-de-problemas-de-saude-oms-lanca-a-campanha-vamosconversar\&Itemid=839> acessado em: 07 de Junho de 2017 
SAVIANI-ZEOTI, Fernanda; PETEAN, Eucia Beatriz Lopes. Apego materno-fetal, ansiedade e depressão em gestantes com gravidez normal e de risco: estudo comparativo. Estud. psicol. (Campinas), Campinas, v. 32, n. 4, p. 675-683, Dec. 2015 Available from <http://www.scielo.br/scielo.php? script=sci_arttext\&pid=S010366X2015000400675\&Ing=en\&nrm=iso>. access on 26 Abril 2017. http://dx.doi.org/10.1590/0103-166X2015000400010.

Gauthreaux C.; Negron J.; Castellanos D.; Ward-Peterson M.; Castro G.; Rodríguez de la Vega P.; Acuña J.M. Associação entre a adoção da gravidez e a experimentação de sintomas de depressão pós-parto entre novas mães nos Estados Unidos. Medicine (Baltimore). 2017 Feb;96(6):e5851. doi: 10.1097/MD.0000000000005851. Disponível em: <htpp://www.ncbi.nlm.nih.gov/pubmed/28178128> acessado em agosto de 2017.

Chojenta CL, Lucke JC, Forder PM, Loxton DJ.Fatores de saúde materna como riscos para a depressão pós-natal: um estudo longitudinal prospectivo. PLoS One. 2016 Jan 19;11(1):e0147246. doi: 10.1371/journal.pone.0147246. eCollection 2016. Disponivel em: <htpp://www.ncbi.nlm.nih.gov/pubmed/26785131> acessado em agosto de 2017

Turkcapar, A.F; Kadıoğlu, N.; Aslan, E.; Tunc, S.; Zayıfoğlu, M.; Mollamahmutoğlu L. Sociodemographic and clinical features of postpartum depression among Turkish women: a prospective study. BMC Pregnancy Childbirth. 2015 May 3;15:108. doi: 10.1186/s12884-015-0532-1. em <htpp://www.ncbi.nlm.nih.gov/pubmed/25935726> Acesso em 02 agosto de 2017.

Markhus MW, Skotheim S, Graff IE, Frøyland L, Braarud HC, Stormark KM, Malde MK. O índice de ômega-3 baixo na gravidez é um fator de risco biológico para a depressão pós-parto. PLoS One. 2013 Jul 3;8(7):e67617. doi: 10.1371/journal.pone.0067617. Print 2013. Disponível em: <htpp://www.ncbi.nlm.nih.gov/pubmed/23844041> Acesso em 02 de agosto de 2017. 